\title{
Implementation of a High-Repetition-Rate Laser in an AP-SMALDI MSI System for Enhanced Measurement Performance
}

\author{
Max A. Müller ${ }^{1}$, Mario Kompauer ${ }^{1}$, Kerstin Strupat ${ }^{2}$, Sven Heiles ${ }^{1}$, Bernhard Spengler ${ }^{1 *}$
}

${ }^{1}$ Institute of Inorganic and Analytical Chemistry, Analytical Chemistry, Justus Liebig University Giessen, 35392 Giessen, Germany

${ }^{2}$ Thermo Fisher Scientific (Bremen) GmbH, 28199 Bremen, Germany

\section{Supporting information}

\section{Table of contents}

1. Microscopic image of laser ablation spots in "spot mode" on red dye

2. Microscopic image of laser ablation spots in "full-pixel mode" on red dye S3

3. Total ion charge at different scan speeds on mouse brain tissue S3

4. Mass spectra from mouse brain tissue at different scan speeds $\quad$ S4

5. Number of detected phospholipid signals on mouse brain tissue at different scan speeds S4

6. Pixel coverage for selected signals in MALDI MS images at different scan speeds $\quad$ S5

7. High speed MALDI MSI image of mouse brain tissue measured with 28 pixels $/ \mathrm{s} \quad \mathrm{S} 5$

8. High speed MALDI MSI image of mouse brain tissue measured with 41 pixels/s $\quad \mathrm{s} 6$

9. MALDI MS ${ }^{2}$ results for "full-pixel mode" and "spot mode" from mouse brain tissue S6

10. Established protocol for hematoxylin and eosin staining $\quad \mathrm{S7}$

11. Mass deviation for selected signals from mouse brain tissue $\quad$ S7

*Address correspondence to:

Institute of Inorganic and Analytical Chemistry

Justus Liebig University Giessen

Heinrich Buff Ring 17

35392 Giessen, Germany

Phone: +49 6419934801

e-mail: bernhard.spengler@anorg.chemie.uni-giessen.de 


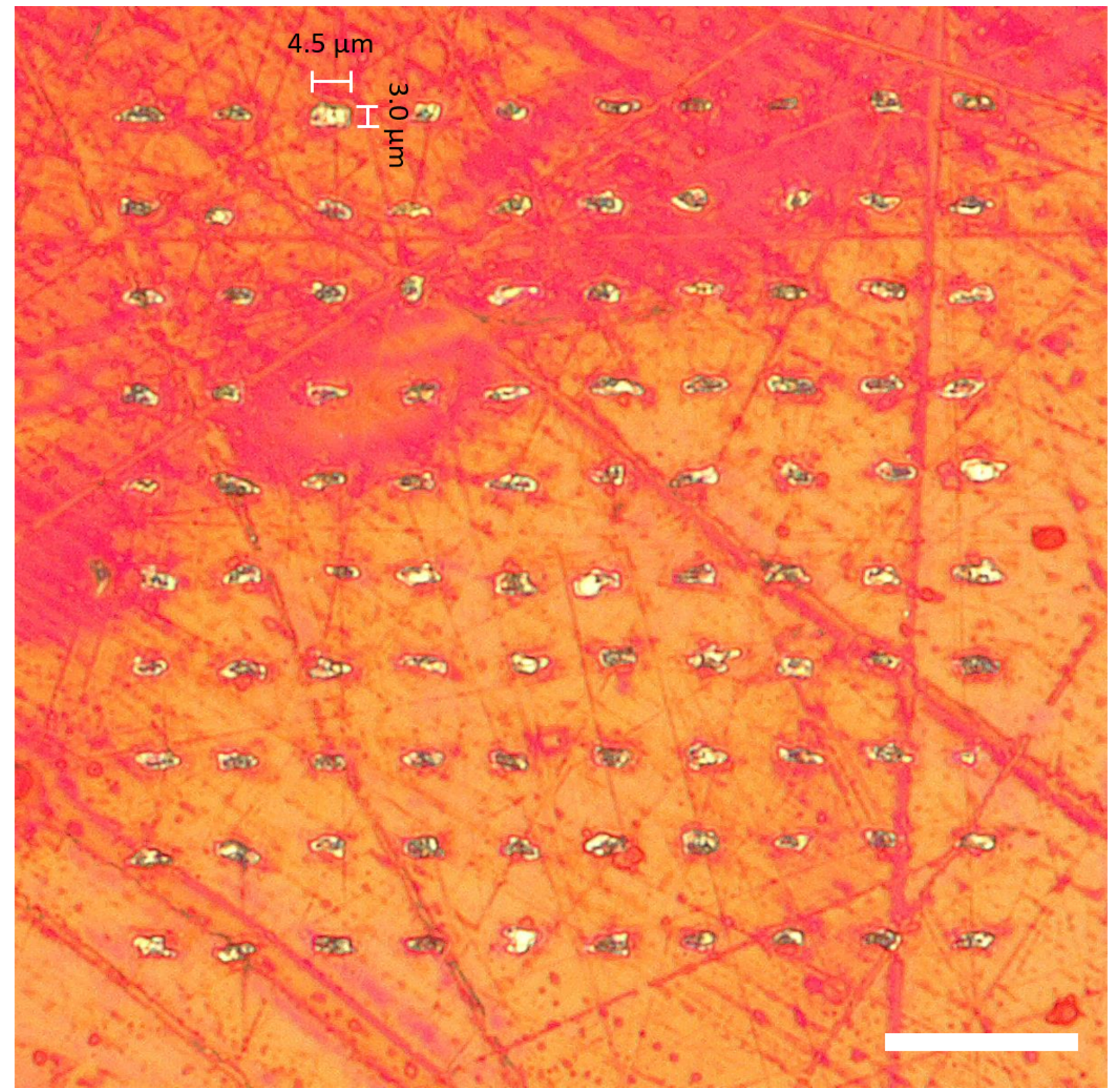

Figure S1. Light microscopic image of the laser ablation pattern in "spot mode" MSI of a red-dye coating. Scale bar: $20 \mu \mathrm{m}$. 


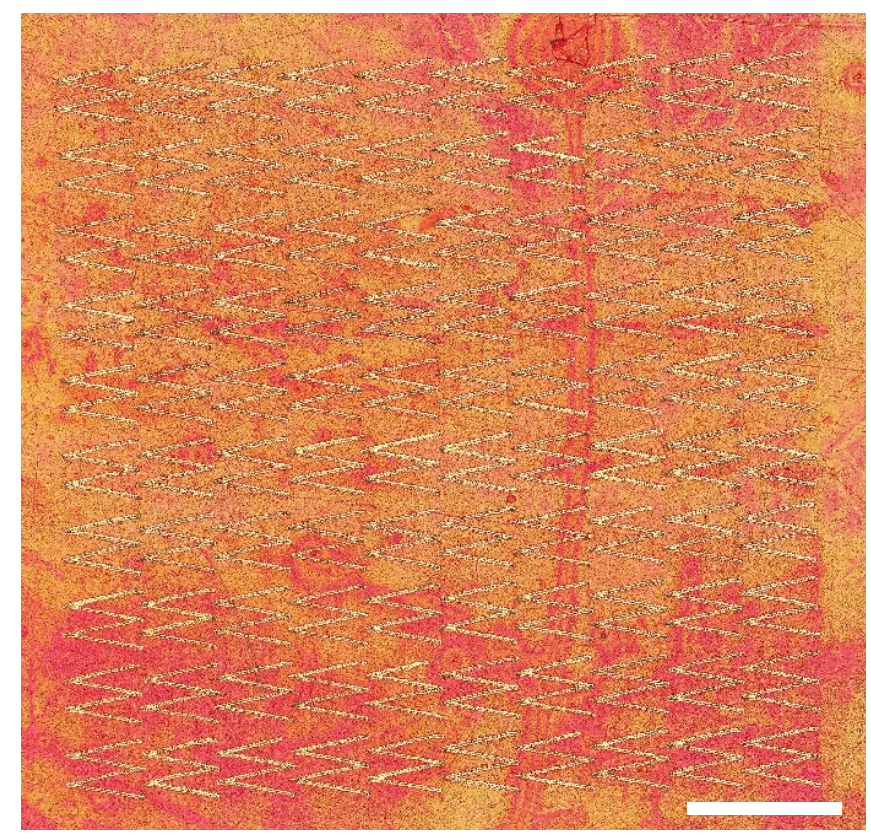

Figure S2. Microscopic image of the laser ablation pattern generated in "full-pixel mode" MSI on red dye for a pixel size of $50 \mu \mathrm{m}$ edge length. Scale bar: $100 \mu \mathrm{m}$.

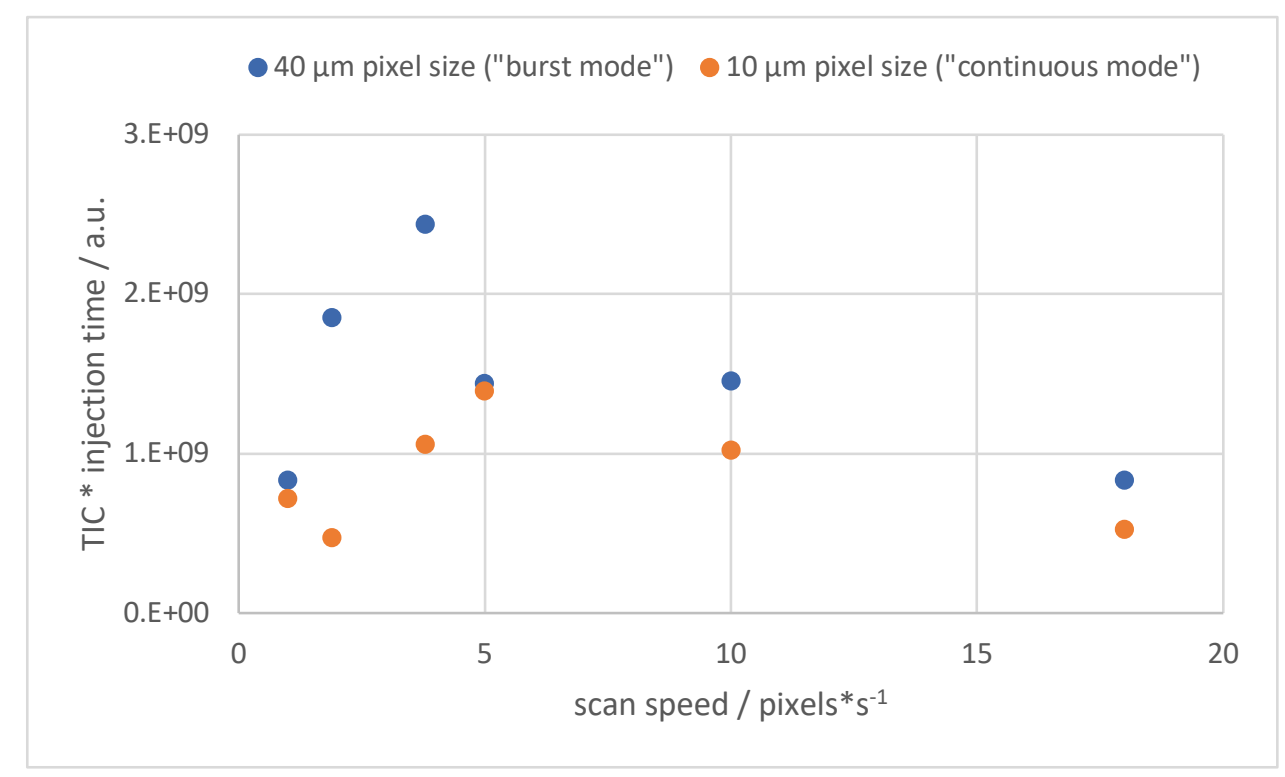

Figure S3. Total ion charge (i.e. total ion current (TIC) times injection time) for different scan speeds in "line scan" modes MALDI MSI on mouse brain tissue at a pixel size of $10 \mu \mathrm{m}$ ("continuous mode", orange dots) and $40 \mu \mathrm{m}$ ("burst mode", blue dots). "Spot mode" MALDI MSI is included at 1 pixel/s. The $m / z$ range was set to $m / z 600-1000$. Laser focus diameter was $5 \mu \mathrm{m}$ in all cases. 


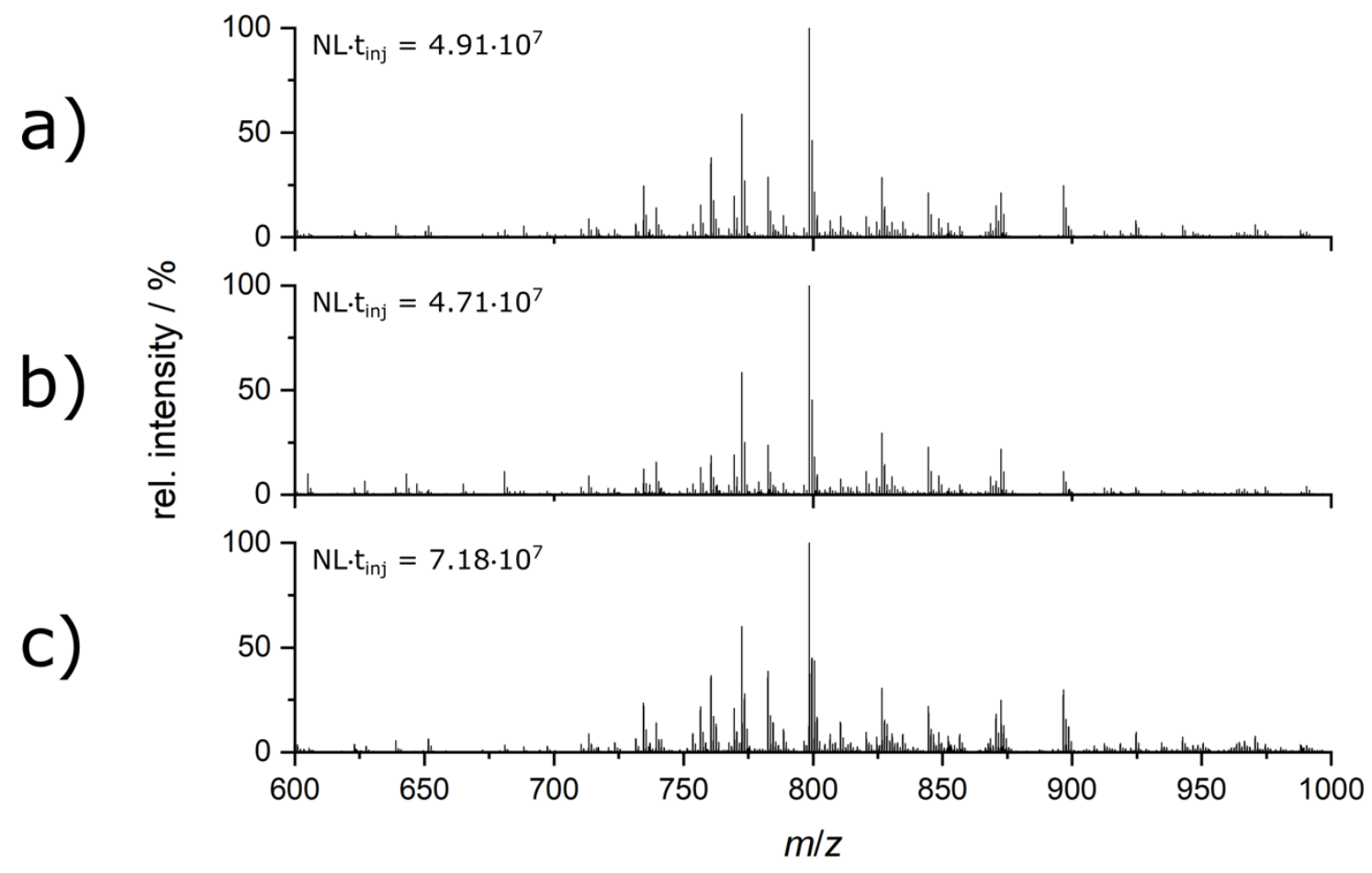

Figure S4. Mass spectra (100 summed) from DHB-coated mouse brain tissue at $10 \mu \mathrm{m}$ pixel resolution. a) "Spot mode", $R=240,000, \mathrm{~T}_{\text {inj }}=500 \mathrm{~ms}, \mathrm{NL}=9.81^{*} 10^{4}$; b) 1.9 pixels $/ \mathrm{s}$ "continuous mode", $\mathrm{R}=240,000, \mathrm{~T}_{\text {inj }}=500 \mathrm{~ms}, \mathrm{NL}=9.42^{*} 10^{4}$; c) 18 pixels $/ \mathrm{s}$ "continuous mode", $\mathrm{R}=15,000, \mathrm{~T}_{\text {inj }}=40 \mathrm{~ms}$, $\mathrm{NL}=1.79 * 10^{6}$.

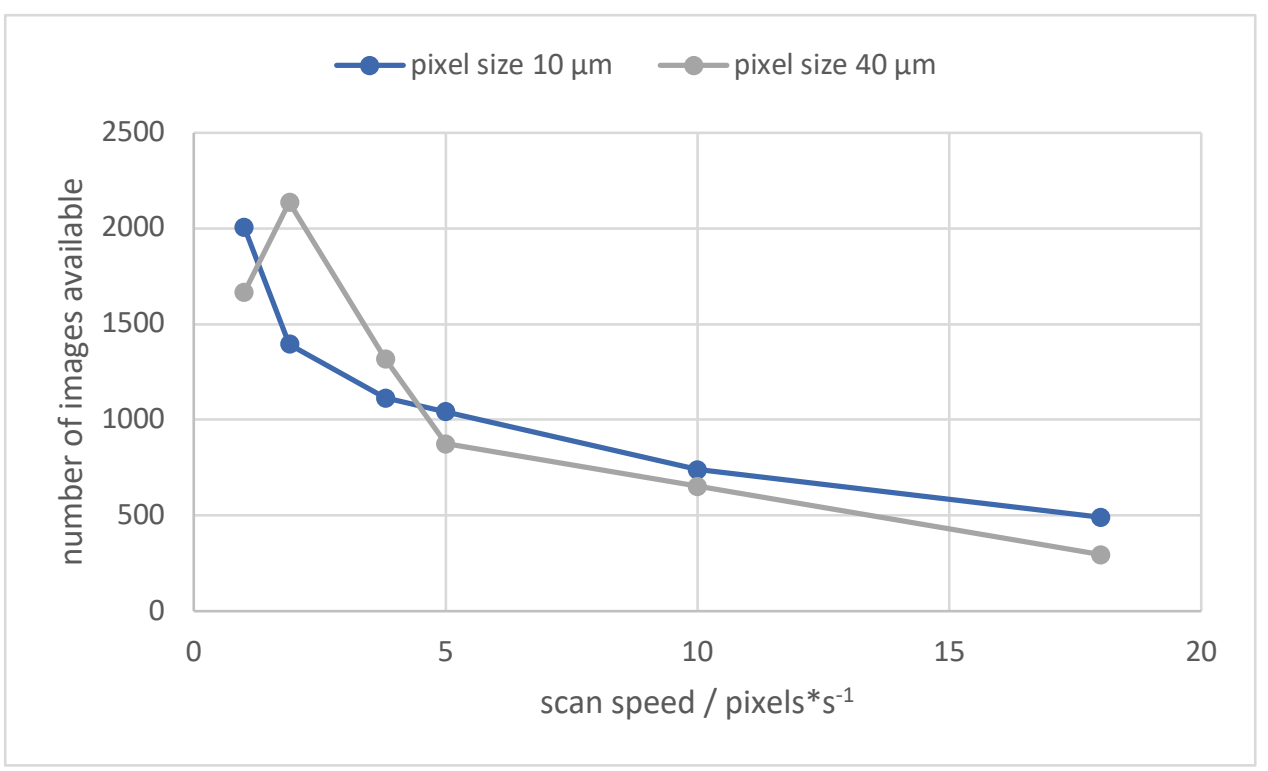

Figure S5. Number of detected and imaged phospholipid signals, measured in "line scan" MALDI-MSI experiments from DHB-coated mouse brain tissue sections with a pixel coverage of $12.5 \%$ or higher as a function of scan speed. "Spot mode" is included at a speed of 1 pixel/s. Blue dots: pixel size $10 \mu \mathrm{m}$ ("continuous mode"), grey dots: pixel size $40 \mu \mathrm{m}$ ("burst mode"). 


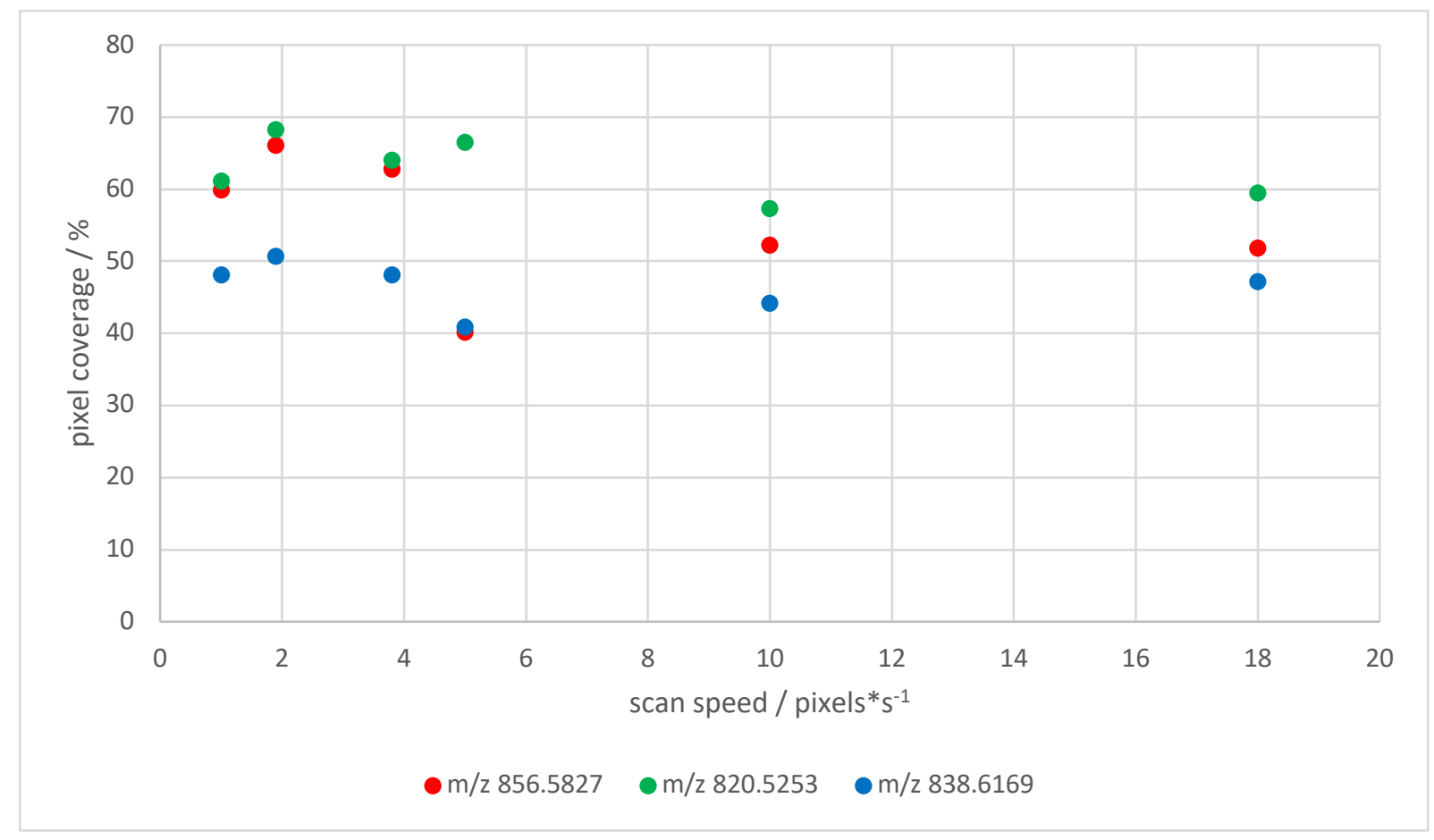

Figure S6. Pixel coverage of three selected $\mathrm{m} / \mathrm{z}$ values for measurements in spot mode (1 pixel/s) and "burst mode" at different scan speeds, as shown in Figure 2a-f.
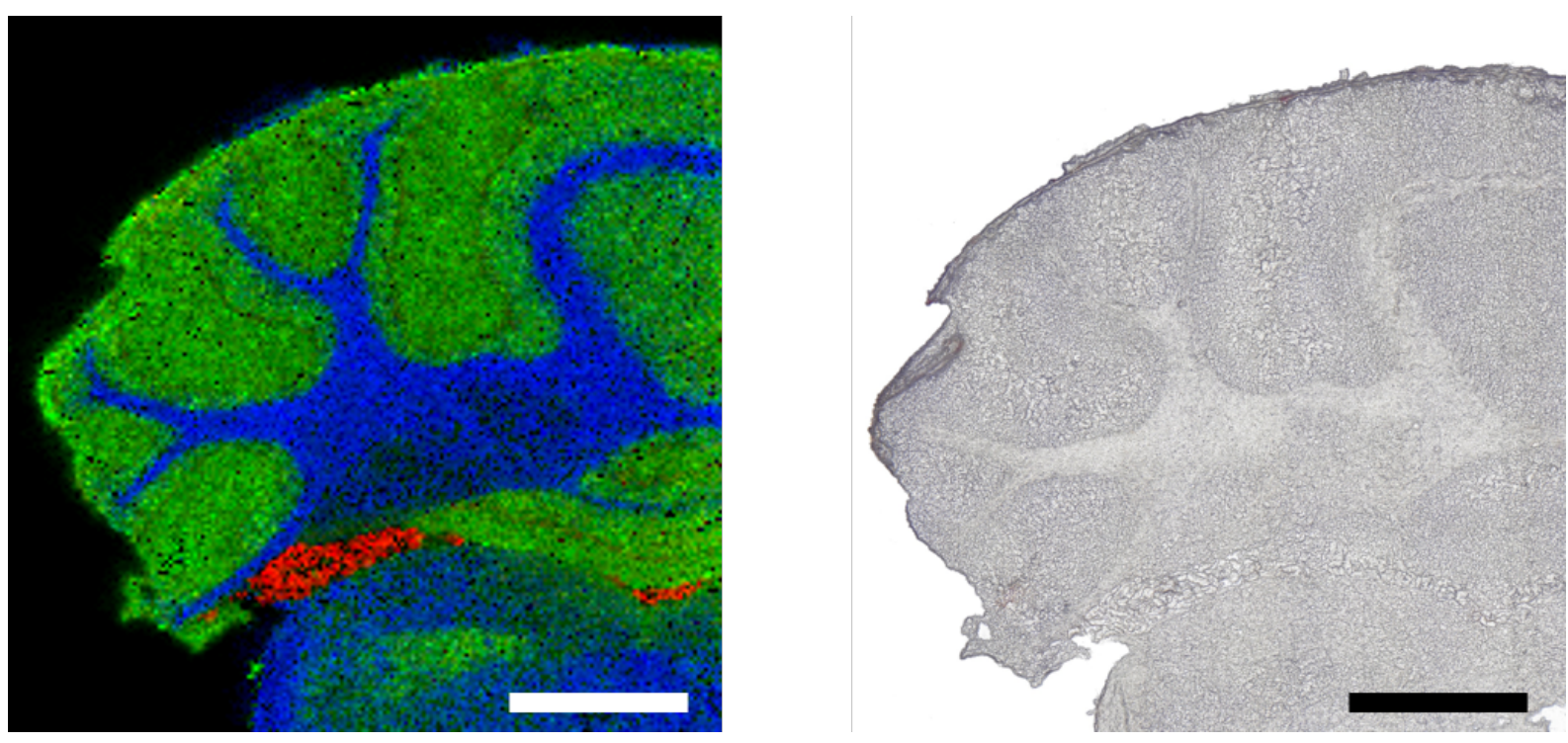

Figure S7. High-speed measurement with $\mathbf{2 8}$ pixels/s on a $\mathbf{Q}$ Exactive HF-X instrument at a mass resolution of 15,000@ m/z 200 and an injection time of 21 ms. MALDI MSI ( $200 \times 200$ pixels) of mouse brain cerebellum measured in continuous mode at a pixel size of $20 \mu \mathrm{m}$, with no normalization being applied. Measurement time was 24 minutes. Color coding: red: $m / z$ 848.55425, [PC 38:4 + K] $]^{+}$; green: $\mathrm{m} / \mathrm{z}$ 772.52499, [PC 32:0 + K] $]^{+}$; blue: $\mathrm{m} / z$ 866.64538, [PI-Cer d40:0 + H] $]^{+}$. The scale bar corresponds to $1 \mathrm{~mm}$. 

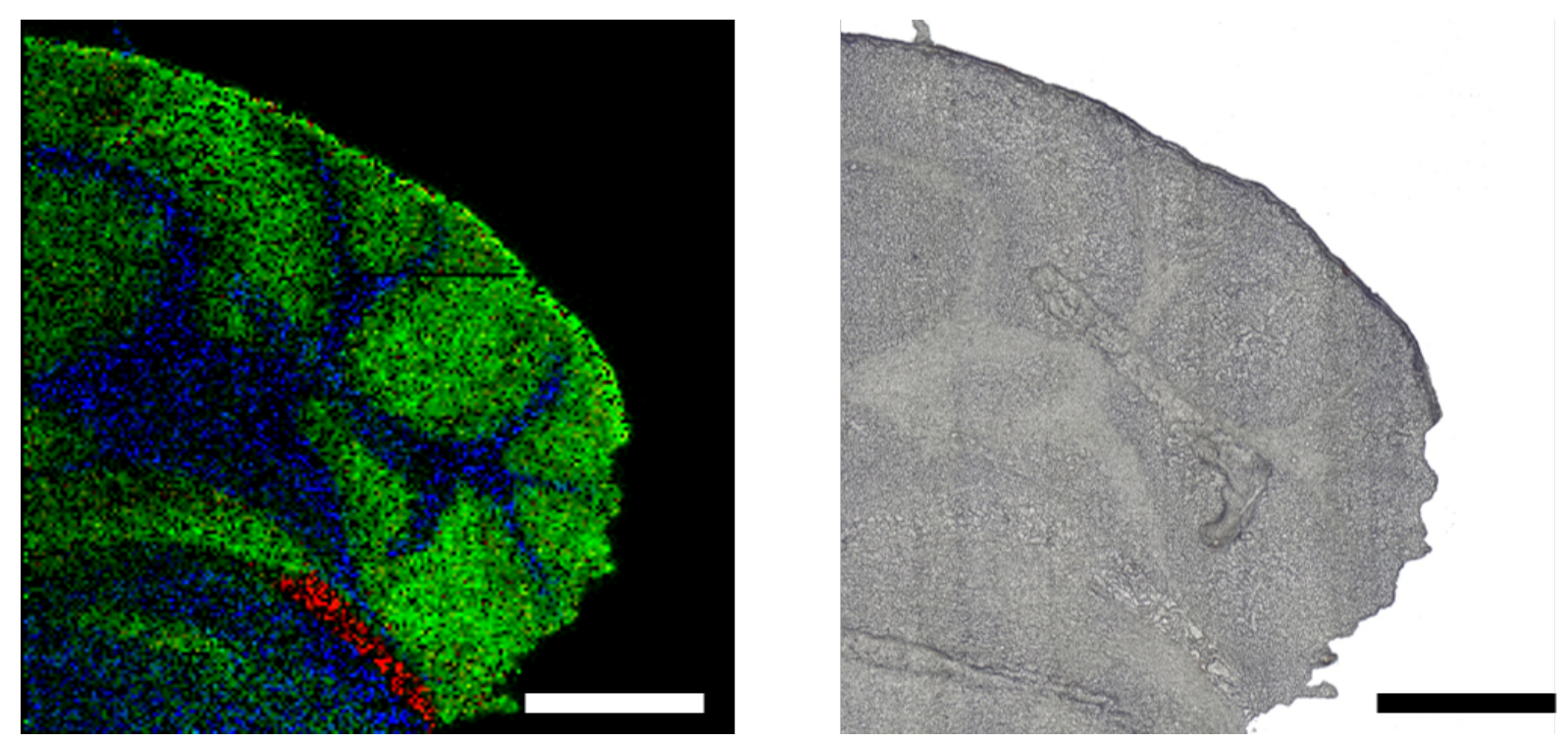

Figure S8. High-speed measurement with $\mathbf{4 1}$ pixels/s on a Q Exactive HF-X instrument at a mass resolution of 7,500@ m/z 200 and an injection time of 13 ms. MALDI MSI ( $200 \times 200$ pixels) of mouse brain cerebellum measured in continuous mode at a pixel size of $20 \mu \mathrm{m}$, with no normalization being applied. Measurement time was 16 minutes. Color coding: red: $m / z$ 848.55508, [PC 38:4 $+\mathrm{K}]^{+}$; green: $\mathrm{m} / \mathrm{z}$ 772.52441, [PC 32:0 + K] $]^{+}$); blue: $\mathrm{m} / \mathrm{z}$ 838.61525, [PI-Cer d38:0 $\left.+\mathrm{H}\right]^{+}$. The scale bar corresponds to $1 \mathrm{~mm}$.

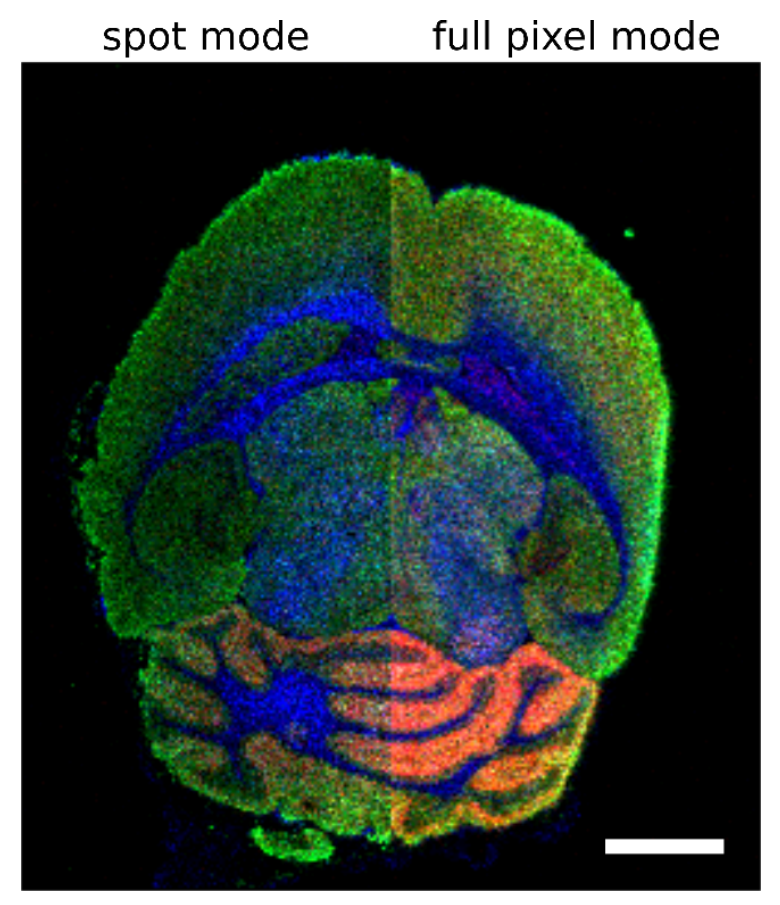

Figure S9. MALDI MS'I image of a horizontal mouse brain section measured partly with "spot mode" and "full-pixel mode", respectively, with no normalization being applied. Isolation of $\mathrm{m} / \mathrm{z} 814.6 \pm 0.5$ was performed and multiple nearly isobaric phospholipid species were assigned based on their fragmentation pattern. Color coding: red: $m / z 162.9562$ (headgroup of [PC 40:6 - $\left.\mathrm{NMe}_{3}\left(+{ }^{13} \mathrm{C}_{1}\right)+\mathrm{K}\right]^{+}$), green: $m / z 673.5174$ (headgroup loss of [PE 40:6 + Na] ${ }^{+}$), blue: $m / z 184.0739$ (headgroup of $\mathrm{PC} 38: 2+\mathrm{H}]^{+}$). Also, [PE O-40:7 + K] $]^{+}$was identified as a co-isolating phospholipid, but not shown here. Scale bar: $2 \mathrm{~mm}$. 
Table S1. Established protocol for hematoxylin and eosin staining by submerging tissue in solvents.

\begin{tabular}{|l|l|}
\hline Solvent & Duration \\
\hline $100 \%$ ethanol & $2 \mathrm{~min}$ \\
\hline $70 \%$ ethanol & $2 \mathrm{~min}$ \\
\hline $40 \%$ ethanol & $2 \mathrm{~min}$ \\
\hline Aqua dest. & $2 \mathrm{~min}$ \\
\hline Hematoxylin & $12 \mathrm{~min}$ \\
\hline Tap water & $10 \mathrm{~min}$ \\
\hline $1 \%$ eosin $Y$ & $1 \mathrm{~min}$ \\
\hline Aqua dest. & $2 \mathrm{~min}$ \\
\hline $40 \%$ ethanol & $2 \mathrm{~min}$ \\
\hline $70 \%$ ethanol & $2 \mathrm{~min}$ \\
\hline $100 \%$ ethanol & $2 \mathrm{~min}$ \\
\hline Xylene & $2 \mathrm{~min}$ \\
\hline
\end{tabular}

Table S2. Mass deviation of signals displayed in Figure 2 from theoretical $m / z$ values at different scan speeds. Mass accuracy is usually better than $0.5 \mathrm{ppm}$ using lock mass correction. For measurements at high speed and reduced mass resolution, larger mass deviations are observed due to overlap and merge of signals in complex samples.

\begin{tabular}{|c|c|c|c|c|c|c|}
\hline $\mathrm{m} / \mathrm{z}_{\text {calculated }}$ & \multicolumn{2}{|c|}{856.5827} & \multicolumn{2}{|c|}{820.5253} & \multicolumn{2}{|c|}{838.6169} \\
\hline $\begin{array}{l}\text { Scan speed/ } \\
\text { pixels*s }{ }^{-1}\end{array}$ & $\mathrm{~m} / \mathrm{z}$ & $\Delta / \mathrm{ppm}$ & $m / z$ & $\Delta / \mathrm{ppm}$ & $m / z$ & $\Delta / \mathrm{ppm}$ \\
\hline Spot mode & 856.5825 & -0.2 & 820.5253 & $<0.1$ & 838.6168 & -0.1 \\
\hline 1.9 & 856.5825 & -0.2 & 820.5253 & $<0.1$ & 838.6169 & $<0.1$ \\
\hline 3.8 & 856.5829 & 0.2 & 820.5254 & 0.1 & 838.6164 & -0.6 \\
\hline 5 & 856,5809 & $-2,1$ & 820,5254 & 0.1 & 838,6171 & 0.2 \\
\hline 10 & 856.5833 & 0.7 & 820.5264 & 1.3 & 838.6162 & -0.8 \\
\hline 18 & 856.5906 & 9.3 & 820.5249 & -0.5 & 838.6222 & 6.4 \\
\hline$m / z$ calculated & \multicolumn{2}{|c|}{844.5253} & \multicolumn{2}{|c|}{832.6637} & & \\
\hline $\begin{array}{l}\text { Scan speed/ } \\
\text { pixels* } \text { s }^{-1}\end{array}$ & $\mathrm{~m} / \mathrm{z}$ & $\Delta / \mathrm{ppm}$ & $\mathrm{m} / \mathrm{z}$ & $\Delta / \mathrm{ppm}$ & & \\
\hline Spot mode & 844.5253 & $<0.1$ & 832.6637 & $<0.1$ & & \\
\hline 1.9 & 844.5252 & -0.1 & 832.6635 & -0.3 & & \\
\hline 3.8 & 844.5254 & 0.1 & 832.6635 & -0.2 & & \\
\hline 5 & 844.5254 & 0.1 & 832.6646 & 1.0 & & \\
\hline 10 & 844.5280 & 3.1 & 832.6627 & -1.1 & & \\
\hline 18 & 844.5262 & 1.0 & 832.6652 & 1.8 & & \\
\hline
\end{tabular}

